\title{
La transparencia contable de la gestión
}

\author{
María Eugenia De la Rosa Leal ${ }^{14}$
}

México

\section{Resumen}

Este trabajo realiza un análisis de las posturas teóricas de las variables gestión y contabilidad como parte de la función de gobierno en las organizaciones, en consecuencia una de la otra y su correspondencia en la transparencia contable. Se realiza una contrastación metodológica semántica de la gestión, la contabilidad y la transparencia con el propósito de tipificarlas en sus condiciones y realidades nacionales en México. Se utilizó para el estudio tres variables de información de los estados contables y su valor ponderado, en una muestra de estados financieros del ejercicio 2012 publicados en la página web de organizaciones económicas industriales.

Palabras clave: gestión, organizaciones, transparencia contable.

\begin{abstract}
This paper analyses the theoretical positions of management and accounting variables as a part of the role of the government in organizations, one as a consequence of the other and their correspondence in accounting transparency. A methodological semantics contrast of management, accounting and transparency is performed with the purpose of standardizing them in their national conditions and realities in Mexico. Three information variables of accounting statements and their weighted values were used in this paper, in a sample of financial statements from 2012 that were published in the industrial organizations webpage.
\end{abstract}

Key words: management, organizations, accounting transparency

${ }^{14}$ Profesora Universidad de Sonora (México).Email: maedelarosa@hotmail.com Recibido: 08/07/2014 - Versión final aceptada: 20/04/2015 


\section{Introducción}

Entre las líneas de interpretación de fenómenos de estudio resultantes de la actividad de las organizaciones, la gestión y la contabilidad revelan diversas situaciones, desde la eficiencia, la productividad, hasta el compromiso social y financiero. En este sentido, para estudiar la gestión es necesario entender el funcionamiento de las organizaciones y sus contextos que influyen y modelan su cultura y comportamiento.

Históricamente, las organizaciones como actores económicos, participan activamente teniendo sobre sí la exigencia de ser efectivas y apoyar en la demanda laboral y el ciclo de consumo, ayudando a la vez a una nivelación económica y la adopción de tecnologías, no sólo de su actividad principal, también de la inmersión en la competencia global y las tecnologías de información.

De forma, que la gestión como suma de decisiones de hacer o no hacer y sus consecuencias presentan diversos estadios de expresión, muchos de los cuales son difíciles de establecer con objetividad o de interiorizar en términos monetarios, provocando que la contabilidad como instrumento de la administración para el control y registro de actividades económicas con efecto monetario tenga problemas para captarlo. A la vez que puede confundir el reconocimiento contable, su devengación y período de realización, contradiciendo su calidad informativa en la toma de decisiones directivas.

La importancia teórica de la contabilidad estriba entonces, en la capacidad para valuar, presentar y revelar la traducción de los actos de gestión en conceptos contables patrimoniales y operativos, contribuyendo a la transparencia de la situación real y su impacto hacia dentro en la generación de recursos y utilidades, en un acto de responsabilidad de la gobernanza con sus inversores y distintos interesados. Vista esta transparencia como la necesidad de conocer con detalle y claridad las formas de inversión y los resultados logrados en la operación de la organización.

Por tanto, la contabilidad posee el atributo de dirigir la información que se procesa hacia la necesidad de los usuarios como enfoque de atención, entendiendo que la información contable toma validez e importancia en la medida que cumple con las características, contenido 
y detalle de la información que son significativos para el o los usuarios, aceptándose con esto la existencia del paradigma del usuario conforme lo establece Tua (1990), quién defiende la idea de la cientificidad de la contabilidad, a pesar y a la par del desarrollo normativo y conceptual de la normas y criterios contables en la globalización y armonización de la información financiera de las organizaciones.

Este trabajo, defiende esta posición de cientificidad, sin embargo no es razón del mismo argumentar a la contabilidad como una ciencia de estudio, orientando este comentario a abrir la posición de estudio de la misma con un enfoque más allá de un procedimiento o una técnica.

La propuesta teórica de abordaje del trabajo es a partir de la función de gestión como hilo conductor, a fin de descubrir si la transparencia contable logra reflejar la imagen fiel de la organización y el efecto de su gestión, entendiendo que la gobernanza de las organizaciones es ejercida desde la dirección, y su efecto causal es captado por los canales formales y registrado por el sistema contable que vincula y comunica a la organización.

Es importante establecer que el sistema contable de cada organización, se diseña en razón de su actividad principal, documentando y confirmando que las operaciones con efecto financiero correspondan a la misma y a su vez delaten con claridad la situación de la actividad realizada, sea esta de origen interno, por intercambio con terceros o consecuencia de un evento externo que afecte las fuentes y recursos de la organización.

La misión principal de la contabilidad es entonces, lograr una comunicación de los efectos económicos y financieros hacia el interior de la organización, supervisando y controlando la evidencia de los hechos para lograr que los estados contables presenten en forma adecuada, accesible y oportuna el resultado financiero, ingresos y gastos originados, generando una historia y tendencias de trabajo de la gestión y sus decisiones que permite evaluar y tomar decisiones en pro de la productividad y eficiencia económica de la organización.

Este trabajo, estudia la revelación efectiva de los distintos aspectos financieros, a través de los estados contable, tratando de responder a ¿la transparencia informativa de los estados contables permite cumplir con la misión de la contabilidad?, para lo cual se aplicó un estudio do- 
cumental a los estados financieros de usos social, publicados en páginas web o en periódicos de circulación periódica en México, durante el año 2012, considerando dos premisas: el uso de normas contables para la uniformidad de la presentación y la relación con aspectos financieros de operación, conforme los subsistemas internos de origen y aplicación de recursos, tomando como muestra sólo aquellos estados contables que presentaban por lo menos el balance general y el estado de resultados del mismo período.

El desarrollo del trabajo, inicia con los antecedentes teóricos principales de la gestión y de la contabilidad, se ubica el campo y cultura organización mediante la revisión de las teorías organizacionales estableciendo la influencia teórica que ambas han recibido en su relación participativa, concluyendo con la integración del paradigma del usuario en la interpretación del estudio.

Este trabajo, sólo estudia la gestión directiva, y no atiende otros campos de actuación de la gestión como los recursos humanos, el capital intelectual, el riesgo, la calidad o el conocimiento.

\section{Antecedentes}

El estudio de las organizaciones como actores económicos, las ubica como un poderoso instrumento social que evalúa y busca alcanzar sus objetivos a través de la coordinación de acciones, líderes, especialistas, obreros y materias (Etzioni, 1967 citado por Córdova, Font, Gudiño, Hernández y Sánchez, 1998). En este protagonismo, las organizaciones se interrelacionan con otras similares con características particulares de independencia y especialidad integrando una red de relaciones organizacionales colectiva que retoma la importancia de la actividad humana y multiplica en su colectividad la suma de visiones individuales.

Las organizaciones, han sido vistas como estructuras complejas y en este sentido estudiadas y entendidas como: máquina, cerebro, sistema político, sistema de dominación o un sistema cultural (Córdova et al, 1998). En ellas el sentido de la eficiencia y la calidad, han introducido procesos de decisión casi inmediatos (Segundo, 1998) que ponen a prueba la información con que se cuenta y los instrumentos y mecanis- 
mos con que se opera, reflejando la capacidad y tiempo de respuesta de la organización para atender las demandas de su entorno.

De manera que la eficiencia de la organización considera tres situaciones:

- Las necesidades de las organizaciones son diferentes;

- Muchas organizaciones necesitan respuestas inmediatas o soluciones a corto plazo; $y$

- Las herramientas cuantitativas que miden la eficiencia son complicadas y con escaso grado de generalidad (Segundo, 1998).

De tal manera, que además de la capacidad de respuesta, la información como sustento debe otorgar los elementos claros y precisos necesarios de evaluación y medición de la gestión, que respaldada en procedimientos e indicadores, revele la realidad operativa y valore los sistemas primarios de operación como la producción, la flexibilidad de los bienes y servicios, e incluso el efecto de los mercados financieros nacionales

Por otro lado, si la organización tiene una participación dinámica en la competencia global, la gestión de la eficiencia por intervención en las debilidades y oportunidades exige un cambio, adaptación y/o transformación interna para alcanzar las metas a corto y largo plazo (Segundo, 1998), por lo que la medición de la eficiencia es una guía útil para los gestores, siendo ésta el terreno de la contabilidad.

Al fijarse las metas y objetivos de la organización, es necesario un sistema de evaluación y medición de logros y desviaciones, que funcione como sistema de control de la gestión, e incorpore a los directivos de la organización como centros o áreas de responsabilidad. Este sistema de control genera, a su vez, un sistema de información en donde la contabilidad juega un papel importante por su capacidad para captar las acciones, logros y conflictos exteriorizados y traducirlos en indicadores cuantitativos y datos cualitativos significativos.

De manera, que la contabilidad interviene como un sistema de control formal de información y medición dirigido a los indicadores financieros para: 
- Establecer estándares y puntos críticos

- Medir el desempeño, y

- Corregir desviaciones (Koontz 1994: 128)

En el modelo contable anglosajón -utilizado por México- la contabilidad de indicadores económicos financieros se reconoce como contabilidad financiera, diferenciándola de la contabilidad de costos, contabilidad administrativa y contabilidad fiscal, por el grado de especialización, enfoque de uso y subordinación de cada una de ellas a la financiera. En contraste, el modelo continental utilizado en Europa distingue a la contabilidad como un sistema de información estatizado que utiliza un plan de cuentas nacional establecido por las autoridades fiscalizadoras. Este modelo distingue a la contabilidad de gestión, como la contabilidad directiva, concibiéndola con múltiples funciones a partir del control de los costos.

En ese contexto, la contabilidad de gestión es un sistema de información formalizada que partiendo de un proceso planificador -presupuestos- y un proceso de cálculo y evaluación de desviaciones, estructura a la organización en centros con objetivos fijados que coinciden en el logro del objetivo principal, evaluando su cooperación participativa en los resultados de la organización a través de los costos y sus desviaciones (AECA, s/f).

Mientras en México, la contabilidad financiera se orienta por las Normas de Información Financiera (NIF) para producir información financiera de las actividades económicas de la organización, reconociendo como fuentes de las mismas las transformaciones internas de los recursos, transacciones con terceros y eventos externos que afecten la estructura de los recursos o sus obligaciones (CINIF, 2014: NIF A1, 15), para posteriormente involucrarla en la evaluación, interpretación $\mathrm{y}$ toma de decisiones.

Por otra parte, la situación cultural en las organizaciones, sistemas administrativos y modelos contables mexicanos, ha sido adoptar las teorías anglosajonas como propias conllevado el uso de textos y teorías que no siempre han respondido a la realidad de los negocios en México, dando lugar a una apropiación del conocimiento con interpretaciones variadas y tropicalizadas, fuertemente influida por el acceso cercano a 
Norteamérica, situación que ha generado un impacto en el desarrollo y conocimiento contable del país y ha impedido una reflexión introspectiva en su encomienda patrimonial y de información de la gestión económica de la organización, asumiendo modelos extranjeros, que necesitan ser aprendidos, adaptados y aplicados en corto plazo, conllevando consecuentes conflictos de adopción y capacidad de respuesta de la organización (De la Rosa, 2009; Hannan y Freeman , 1992 citado por Shafritz y Steven, 2002: 330-332).

Sumemos a la información interna de la gestión, la importancia del papel socioeconómico de las organizaciones su compromiso patrimonial, imagen social, compromiso comunitario y ambiental. Lo cual implica la existencia de valores y el reconocimiento de un riesgo operativo en la ingeniería de producción, que impacta más allá de sus instalaciones, con una visión de transparencia y responsabilidad social que orienta hacia la necesidad de una contabilidad social de la organización (De la Rosa, 2008; Pahlen y Campo, 2008), reforzando la imagen de transparencia informativa con usuarios potenciales más allá de la gestión.

\section{Acerca de la gestión}

Para cumplir con sus objetivos y actividades necesarias, la organización genera un proceso dinámico de gestión que interactúa con su entorno en un tiempo y contexto específico, con una estructura de cuatro ejes principales: comercial, operaciones, recursos humanos y finanzas, enlazados en un plan integral de sistemas de procesos administrativos, de dirección, planeación, ejecución y control (Koontz y Weihrich, 1994).

Este plan de gestión, actúa de forma paralela con la dirección con un estilo de control administrativo, agregando valor a los productos, bienes o servicios. Su objetivo es implementar sistemas de control adhoc que atiendan el desempeño y soporten el ejercicio de la estrategia, a través de: la planeación estratégica, asignación presupuestal de recursos, medición del desempeño, designación de centros de responsabilidad y fijación de precios de transferencia (Anthony y Govindarajan, 2007: $1-5)$. 
Por su parte, la gestión sobre las finanzas, se asemeja a una red de comunicación de los efectos, en donde, el medio de control responde a las características, necesidades de las estrategias y la forma de operación de las organizaciones. En ella, la información de lo que pasa, es un detector del indicador o norma prestablecida por el medio de control, que ayuda a transformar la retroalimentación del efecto recibido en un comportamiento de la organización hacia lo requerido.

La gestión en sí, es una estrategia, compuesta por un conjunto de sistemas organizativos de intervención como resultado de la adaptación de la organización a su entorno, figura 1.

Figura 1 Sistemas organizativos de gestión

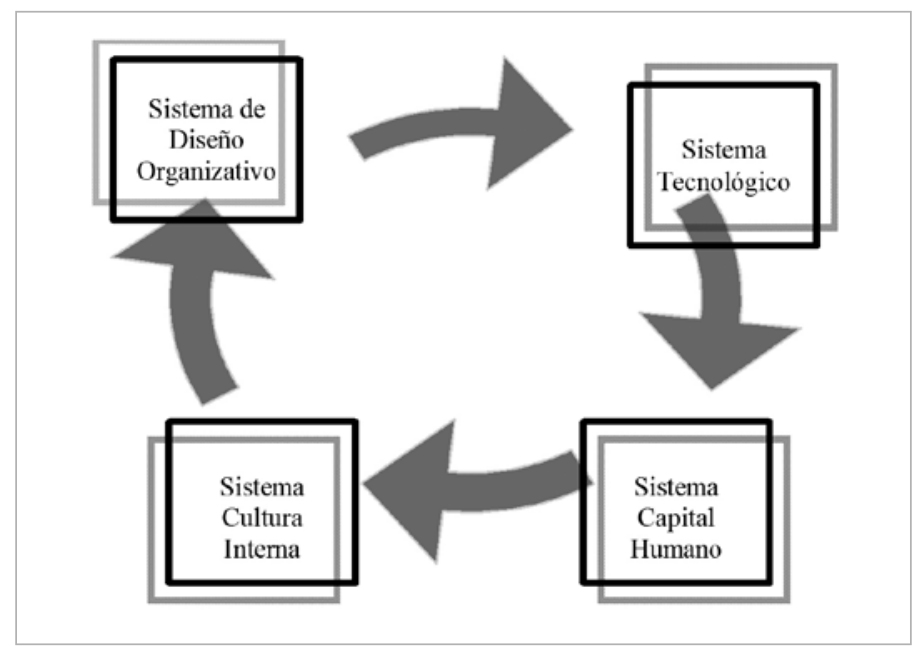

Fuente: Elaboración personal

Estos sistemas de intervención: tecnológico, capital humano, cultura interna y diseño organizativo, actúan como puntales de la estructura de trabajo correlacionando el funcionamiento de operación y el control de la organización, a la vez que influyen en la estrategia general, en sus relaciones con su contexto y en sus decisiones y conductas. 
De alguna manera, la función de gestión emula el ejercicio de gobierno en una organización, dada como un ejercicio de poder y autoridad a través del control y la coerción. Este tipo de gobierno se clasifica de acuerdo a quién ejerce el poder y qué intereses ampara (Giner, Lamo y Torres, 2006: 377). Normalmente estos grupos de control del gobierno son los consejos de administración como decisores del management o gerencia.

Así entonces, la gerencia como actor de la gestión dirige el negocio y asume la responsabilidad de llevar las riendas de la firma con un estilo de arreglo corporativo y jurídico de acuerdo con sus actividades. La gerencia es, por tanto, responsable de gestionar adecuadamente la dirección de las organizaciones (Giner et al, 2006: 370).

En las teorías clásicas de la administración y dirección de empresas, la función y el rol de gerencia es resultado de la creciente burocratización de las organizaciones y del establecimiento de una diferenciación entre la alta dirección, que define políticas, diseña estrategias e innova en sus soluciones, y la gerencia que desarrolla las acciones y contribuye a redefinir y rediseñar los contenidos determinados desde la dirección (Barnard, 1938 citado por Giner et al, 2006: 370).

Las organizaciones necesitan también un control social, orientado en esencia a la observación realista de las desviaciones como una necesidad de orden. Es en esta dimensión, la contabilidad interviene para reconocer y vigilar el logro de las metas económicas convirtiéndose en un mecanismo de información social.

En lo relativo a la gestión, la contabilidad es el ejercicio de identidad de una organización que permite establecer indicadores financieros ad hoc de los principios generales de actuación para todos los miembros de la organización, legitimar la gobernabilidad de la organización y proporcionar un conjunto de estándares del rendimiento organizativo (Hodge, Anthony \& Gales, 1998: 51-55).

Se ha demostrado que la contabilidad provee entre el 46 y el 50\% de la información que la organización necesita para su funcionamiento cotidiano, tanto para usuarios internos como externos, los cuales buscan un conocimiento ético, veraz, suficiente, oportuno, real y creativo de la organización (Moldovan,Achim y Bota, 2010). 


\section{Acerca de la contabilidad}

Como la vida misma, la contabilidad es una continuidad de distintos aspectos de la vida de la organización y apoya la condición legal de existencia en un mundo social y económico, a través de una rendición de cuentas. La contabilidad no funciona autónomamente, lo hace en un marco histórico y cultural que articula el sentido de quién es y qué se hace (Goddard, 2004: 546).

En México, en la medida en que los negocios fueron haciéndose más complejos, se integraron aspectos de control y supervisión que llevaron a la auditoría financiera que surge con la Ley de Títulos y Operaciones de Crédito (1932) lo cual impulsó el protagonismo fiscal y contable como un control público de las organizaciones (De la Rosa y Gracida, 2008).

A la par el surgimiento del modelo capitalista, la creación de bancos y la Ley de títulos y operaciones de crédito permitieron desarrollar aspectos cada vez más complejos de las practicas y la tecnología contable, lo cual transformó a la contabilidad en un sistema de control y lenguaje de comunicación en los negocios (Del Toro, 2003), que en su desarrollo evolutivo doctrinario, separó su estructura de control en los aspectos significativos del negocio, haciendo que los negocios y el registro de sus actividades se tornara cada vez más sofisticado, jugando en ello la intervención de la tecnología, la presencia de nuevos y distintos actores y la aparición de innovadores mecanismos de negociación y financiamiento (De la Rosa y Gracida, 2008), esto modificó el discurso teórico de la contabilidad llevándola de un registro de la memoria económica a un compromiso patrimonial (Pahlen y Campo, 2008: 23-31).

La historia moderna de la contabilidad en México, tiene tres constantes en su evolución y cambio: el contexto de la política económica, la influencia norteamericana y la cambiante política tributaría del país. En donde las exigencias del Tratado de Libre Comercio a partir de 1994 han tenido gran influencia en relación con los vínculos comerciales y la presencia de capitales norteamericanos y la expansión territorial, en donde la tendencia contable nacional ha sido seguir los pasos de nuestro vecino comercial Estados Unidos de Norteamérica, utilizando en el registro de conceptos contables los criterios de evaluación de va- 
lor razonable y exigible, el uso de software y equipos electrónicos y la orientación de la información para fundamentar las decisiones directivas como usuario principal de las organizaciones (De la Rosa, 2009).

Así la percepción de la contabilidad para la rendición de cuentas tiene cuatro dimensiones de intervención:

- Las condiciones causales que como variables culturales, ideológicas, de crisis, de relaciones estructurales y de gobierno, la influyen externamente;

- Las prácticas contables que a partir de prácticas arraigadas, uso de tecnologías y conocimiento financiero, definen la acción e interacción;

- La contabilidad y las prácticas de gobernabilidad basadas en las estructuras de gobierno y las prácticas de indicadores de gestión y otros indicadores; $y$

- Las consecuencias a que se refieren a las prácticas comunes de la contabilidad, la manipulación de la contabilidad, la percepción de las prácticas del ejercicio de la contabilidad, la propensión al cambio y la confrontación de la contabilidad vs la rendición de cuentas (Goddard, 2004: 551).

Por lo que la perspectiva contable de gobernabilidad significa utilizar mecanismos y metodologías con una relación muy cercana a las finanzas, en un contexto y tiempo específico (Brennan y Solomon, 2008: 885-886), de tal forma que la teoría doctrinal contable se une a las finanzas para incorporar conceptos de control y definición de los orígenes y aplicaciones financieras, como una expresión de poder. Con la teoría de costos de transacción (Williamson, 1985, 1986 citado por Brennan y Solomon, 2008), puede afirmarse que la contabilidad permite la gobernabilidad, y una aproximación cuantitativa a las organizaciones.

A partir de lo anterior, si la contabilidad se establece como un mecanismo de evaluación de desempeño en las organizaciones, se otorga a la misma un valor agregado para los accionistas y la administración. Mientras que apoya los propósitos de gestión para cuidar las políticas y su substancia, y ver al poder como una esencia de la gestión, hacia la efectividad y el éxito (Darmer, 2000: 337). 
Sin embargo, en la elaboración de la contabilidad los caminos pueden ser influidos y perder objetividad al existir alternativas a elegir en el registro de políticas de costos, planes de compensación activos y pasivos que impactan de forma diferente los resultados de la gestión, dándose una manipulación contable en una aparente contabilidad creativa, que revela la "verdad" de la organización con un enfoque a conveniencia (Moldova et al., 2010).

De forma que los hábitos, las prácticas de autoridad y el valor de los gerentes son evidenciados en la contabilidad, como un sistema vivo en tensión dominado por el grupo de miembros de la organización que ejercen el poder y la gestión. Situación que la coloca en riesgo de no percibir la problemática y la realidad financiera (Goddard, 2004: 566.567) afectando el razonamiento de la rutina natural de la contabilidad y por tanto su generación de conocimiento de la gestión y la organización.

Por otro lado, los mecanismos de transparencia bajo el formato contable, logran que los estados financieros y las declaraciones alineen a los accionistas y ejecutivos, a la vez que sopesan a las variables de gobernabilidad y transparencia con los sistemas legales (Brennan y Solomon, 2008: 888).

En la actualidad la era de la información ha permitido que la contabilidad capte al detalle todas las operaciones económicas de las organizaciones y se convierta en el mecanismo con el cual se les fiscaliza por ley, se procese la información requerida por el Sistema Financiero Nacional y se dote a la organización de información suficiente -en ocasiones excesiva- para fundamentar sus decisiones presentes y futuras, en un sentido de la interpretación de la transparencia contable.

Por lo tanto, la contabilidad es responsable de obtener y suministrar información cuantitativa de los resultados a la organización y moderar las decisiones que pueden enfrentar sus dimensiones económicas, sociales y ambientales. Mientras a su vez, asume su propia interpretación, interiorización de su responsabilidad y alcance en revelación detalle y significado.

Teóricamente la contabilidad se ha desarrollado a la par de las organizaciones, la tecnología y la política económica -particularmente el aspecto tributario-. Sumando en cada etapa niveles de participación 
social y de competencia cada vez más importantes. Entre ellas se ha acumulado y fortalecido una serie de propuestas y conocimientos sobre la base del paradigma de utilidad (Hendricksen, 1970: 76 citado por Tua, 1990) que establece como verdad aceptada por la comunidad científica contable, que el objetivo original de presentar información a la gerencia y acreedores se amplió incluyendo a los inversionistas y accionistas como usuarios prioritários; por lo tanto es cuestión principal del paradigma el usuario la determinación de las necesidades y las reglas adecuadas para lograr el beneficio verdadero al usuario, bajo dos enfoques contables: financiero y de gestión.

\section{El sistema contable}

La contabilidad actual, está orientada a facilitar información útil, clasificada, analizada, necesaria y requerida, para la toma de decisiones gerenciales, así como para servir de herramienta de control en el manejo de las operaciones de una organización gubernamental o privada. Esto exige una revisión de sus funciones y un replanteo de sus bases, en un sistema funcional de información y retroalimentación. La contabilidad gerencial, está ligada a la dirección científica que comprende además de los costos totales y los resultados financieros, los análisis de la actividad operativa empresarial, tales como la cadena de valores de las actividades interrelacionadas desde la obtención de las materias primas hasta el producto terminado (Gutiérrez, 2005).

Bajo la base de una gestión efectiva, la información contable representa una ventaja competitiva al segmentar a la organización en centros de costos y de ingresos para su control y revelación suficiente, tomando en cuenta el incremento de la flexibilidad operativa de su organización y capacidad de adaptarse con rapidez, a los cambios y paradigmas con el propósito de lograr la mayor eficiencia.

Son cuatro circunstancias que deben considerarse en el diseño del sistema contable: la presión impositiva, las tareas habituales de la organización, la respuesta y seguimiento de inspecciones de autoridad y la incorporación de nuevas tecnologías y metodologías. Es importante tomar en cuenta también que el sistema contable se enfrenta a captar una serie de consecuencias que rebasan y trastocan la capacidad y eficiencia 
de la gestión desviando e incluso discontinuando total o parcialmente la actividad, tales como: la adaptación a procesos de competencia, la carga tributaria, la baja rentabilidad, el posicionamiento en el mercado y las nuevas tecnologías de producción y servicios. De hecho los sistemas de Contabilidad Gerencial, surgieron para proveer información, dar apoyo a la dirección y control de las empresas, y para promover la eficiencia en la organización.

Así, el sistema de gestión contable está dirigido por un modelo básico complementado por un sistema de información bien planeado y diseñado, ofreciendo compatibilidad, control, flexibilidad y una relación aceptable entre costo y beneficio de su funcionamiento y aprovechamiento (Fernández y Muñoz, 1997). El sistema contable de gestión, se diseña por áreas o centros de responsabilidad, identificándose las diferentes líneas o centros con una función comercial, administrativa, o de producción.

Este sistema conlleva tres pasos básicos:

1. Registro de la actividad financiera en la empresa: registro de todo tipo de transacciones que pueden ser expresadas en términos monetarios y notación de otros aspectos que puedan afectarla económicamente.

2. Clasificación de la información: ordenamiento de los datos, en categoría y detalle con orientación al usuario, se incluyen archivos documentales prueba.

3. Resumen de la información: en modelos de información accesibles y claros para la toma de decisiones, sin sacrificar el nivel de revelación y suficiencia informativa.

Este proceso del sistema está relacionado con algo más que la creación de información, ya que involucra considerablemente la comunicación entre quienes la elaboran, interpretan y utilizan en las decisiones.

El sistema contable siempre debe proporcionar la información precisa para la gestión a los encargados y a varios usuarios externos que muestran interés en la organización. La gestión contable posee sus propios objetivos para colaborar con el logro del ejercicio de la gestión general. Este sistema tiene por objetivo predecir el flujo de efectivo, orientar las decisiones en cuanto a las inversiones y créditos; apoyar 
a los administradores en cuanto a la planeación, la organización y la dirección de los negocios relacionados con la actividad; fundamentar la determinación de precios; cubrir las tarifas tributarias; ejercer un cierto control sobre las operaciones económicas; y contribuir en la evaluación de los beneficios o el impacto social de la actividad que desarrolla la organización.

La clave de intervención contable es lograr que la imagen real y la rendición de cuentas exhiban el derecho patrimonial y los bienes o inversiones disponibles, de forma que se incluyan todos los conceptos relacionados y relativos afectados por la gestión de la organización. Entendiendo que la transparencia contable de la gestión es el resultado de las rutinas de reconocimiento, el fenómeno contabilizado, las relaciones estructurales, la cultura y los valores de las prácticas contables en sus dimensiones de intervención, que finalmente dan una connotación a la rendición de cuentas y a la transparencia.

Así entonces, la transparencia contable es la rendición objetiva de cuentas que permite información clara, evidente sin ambigüedades del origen, aplicación, estructura y resultados patrimoniales de las organizaciones, útiles para todos los posibles usuarios de la información en tiempo y forma.

\section{Estudio de transparencia de la información contable}

A fin de conocer la calidad de la transparencia contable de la gestión en México, se aplicó un estudio exploratorio de estados financieros emitidos y publicados en 2012 sitios web por organizaciones lucrativas que operan en el territorio nacional, aceptando en la muestra de estudio sólo a las organizaciones industriales lucrativas que se identificaban claramente y que presentaban el juego de balance general y estado de resultados con sus anexos del mismo período, del total de ciento cuarenta estados financieros, la muestra final fue de cuarenta conjuntos de estados financieros básicos.

La metodología utilizada fue el análisis de textos a fin de establecer los conceptos contables y determinar los grupos de información bajo el enfoque actividad conforme el constructo de estudio, figura 2. 
Figura 2 Constructo de estudio. Transparencia de la gestión contable

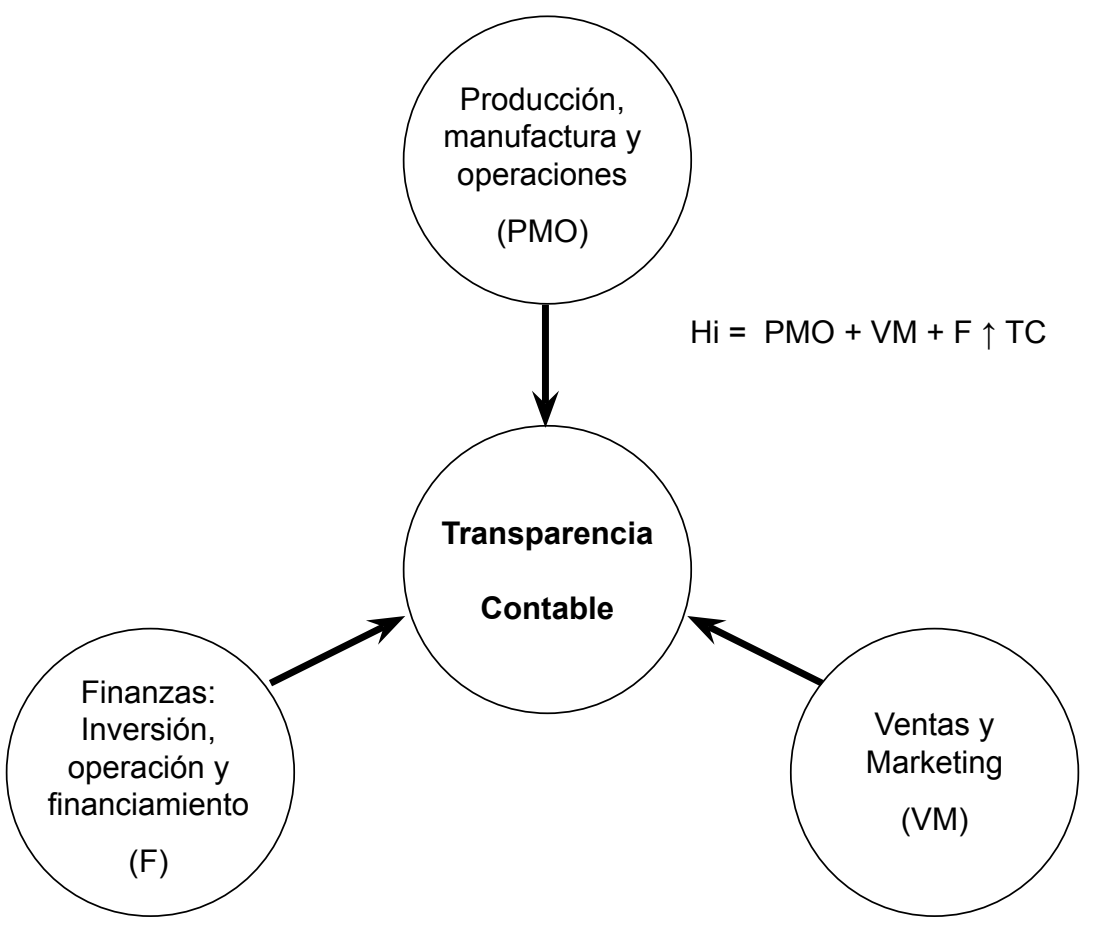

Fuente: Elaboración personal

Las variables de estudio para determinar la transparencia contable de los estados financieros estudiados fueron tres grupos de actividades básicas:

1) Producción, manufactura y operaciones

2) Ventas $y$ Marketing, $y$

3) Finanzas: por actividades de inversión, operación y financiamiento

Como una forma de acercamiento a la información significativa de los resultados de la gestión, agrupando en cada uno de estos grupos los conceptos contables de relación directa y correlacionada conforme 
la técnica contable y de análisis financiero. El objeto de estudio fue la información contable en razón de los puntos críticos.

El alcance del análisis e interpretación de los resultados no utilizó las Normas de Información Financiera, al considerar que estas no son de uso generalizado en los estados contables emitidos en México, no obstante haberse cumplido en 2012 el tiempo firmado de convergencia de estas normas con las internacionales.

La metodología utilizada, permitió determinar un estudio descriptivo de las categorías de los conceptos de la muestra, y aplicar una correlación de Spearman del grado de significación entre conceptos contables teóricamente relacionados.

Quedando como objeto de estudio la información contable, sujeto de estudio las empresas lucrativa, e hipótesis de trabajo que la revelación de los conceptos de: producción, manufactura y operaciones (PMO), los conceptos contables de ventas y marketing (VM); los conceptos contables de finanzas (F) influyen positivamente en el grado de transparencia contable (TC).

$$
\mathrm{Hi}=\mathrm{PMO}+\mathrm{VM}+\mathrm{F} \uparrow \mathrm{TC}
$$

\section{Resultados}

El análisis de la información de los estados contables fue categorizado conforme las dimensiones de los conceptos de información, revisando el grado de revelación y detalle que permitiera otorgar la información necesaria de revelación para un usuario general, se seleccionaron los datos significativos con más de un $10 \%$ del valor del concepto, como criterio de significación.

Los rubros revelados con relación a la información de producción y manufactura, fueron: distribución de productos, detalle del proceso de producción, uso de mano de obra, operación productiva e inventarios, cuya proporción se presenta en el cuadro 1 . 
Cuadro 1. Resultados de la información para la producción, manufactura $u$ operaciones en estados contables emitidos públicamente (2012) en México

\begin{tabular}{|l|l|l|l|}
\hline \multicolumn{1}{|c|}{ Concepto } & \multicolumn{1}{|c|}{$\begin{array}{c}\text { Estatus significativo } \\
\text { en la muestra }\end{array}$} & $\begin{array}{c}\text { Valor } \\
\text { ponderado }\end{array}$ & $\begin{array}{c}\text { Valor total } \\
\text { significativo } \\
\text { del concepto }\end{array}$ \\
\hline \multirow{2}{*}{ Distribución productos } & No da detalle & $44 \%$ & \\
\cline { 2 - 4 } & No aplica & 33 & $77 \%$ \\
\hline \multirow{2}{*}{$\begin{array}{l}\text { Detalle del proceso de } \\
\text { producción }\end{array}$} & No informa & $30 \%$ & \\
\hline \multirow{2}{*}{ Uso de mano de obra } & No da detalle & 30 & $60 \%$ \\
\hline \multirow{2}{*}{ Actividades de operación } & No da detalle & $62 \%$ & \\
\cline { 2 - 4 } & No informa & 37 & $99 \%$ \\
\hline & No da detalle & $36 \%$ & \\
\cline { 2 - 4 } Inventarios & No informa & 27 & $63 \%$ \\
\hline & Aplica pero no informa & 22 & \\
\cline { 2 - 4 } & No aplica & 22 & $77 \%$ \\
\hline
\end{tabular}

Fuente: Elaboración personal del análisis de resultados

Como se observa, la situación dominante en la información de los estados financieros contables es la ausencia de la misma, ya que informa y no da el detalle necesario (en un valor promedio de $41 \%$ ) o no informa (con un valor promedio de 29\%). Así, la muestra estudiada no dota de información necesaria en un $70 \%$. Esta información revelada cumple con informar pero no revela ni permite al usuario general captar la estructura de producción e inventario, ni tampoco permite reconocer el resultado de la gestión.

Con relación a las ventas y marketing, la situación encontrada se analizó mediante la descripción de clientes y la composición de las ventas, cuadro 2. 
Cuadro 2. Resultados de la información para Ventas y Marketing en estados contables emitidos públicamente (2012) en México

\begin{tabular}{|l|l|l|l|}
\hline \multicolumn{1}{|c|}{ Concepto } & $\begin{array}{c}\text { Estatus significativo } \\
\text { en la muestra }\end{array}$ & $\begin{array}{c}\text { Valor } \\
\text { ponderado }\end{array}$ & $\begin{array}{c}\text { Valor total } \\
\text { significativo } \\
\text { del concepto }\end{array}$ \\
\hline \multirow{2}{*}{ Descripción clientes } & No da detalle & $40 \%$ & \\
\cline { 2 - 4 } & No informa & 30 & \\
\hline \multirow{3}{*}{ Composición ventas } & Da detalle limitado & 30 & $100 \%$ \\
\cline { 2 - 5 } & No da detalle & $58 \%$ & \\
\cline { 2 - 4 } & Da detalle & 17 & $75 \%$ \\
\hline
\end{tabular}

Fuente: Elaboración personal del análisis de resultados

La información contable de los conceptos de las acciones de venta y mercadotecnia constituyó un promedio del 87\%; sin embargo, adolecen de detalle, otorgando una descripción baja de las mismas, de manera general no existe una revelación del tipo de mercado de los productos en los estados contables (58\%), la mezcla de conceptos de estrategia de ventas o promoción (30\% y $17 \%$ ) no permite reconocer el tipo de mercado y el peso del mismo para la organización, con relación a la cartera de clientes, se otorga una información limitada del control contable de los clientes con una falta de información en el $30 \%$ de la muestra.

Se observa que en lo que tiene que ver con la generación de ingresos ordinarios, sus costos y gastos relacionados se cuida de no dar información que pueda ser aprovechada por terceros, otorgando información ambigüa.

Con respecto al manejo de las finanzas, con respecto a su origen y aplicación en inversión, operación y financiamiento, la situación encontrada se detalla en el cuadro 3. 
Cuadro 3 Resultados de la información de Finanzas en estados contables emitidos públicamente ( 2012) en México

\begin{tabular}{|l|l|l|l|}
\hline \multirow{4}{*}{ Concepto } & $\begin{array}{c}\text { Estatus significativo } \\
\text { en la muestra }\end{array}$ & $\begin{array}{c}\text { Valor } \\
\text { ponderado }\end{array}$ & $\begin{array}{c}\text { Valor total } \\
\text { significativo } \\
\text { del concepto }\end{array}$ \\
\hline \multirow{4}{*}{ Inversión } & Patrimonio & $21 \%$ & \\
\cline { 2 - 4 } & Activos corrientes & 17 & \\
\cline { 2 - 5 } Operación & Activos fijos & 13 & $51 \%$ \\
\hline \multirow{5}{*}{ Financiamiento } & No da detalle & $36 \%$ & \\
\cline { 2 - 5 } & No informa & 27 & \\
\cline { 2 - 5 } & Gastos cotidianos & 18 & $81 \%$ \\
\hline & No da detalle & $33 \%$ & \\
\cline { 2 - 5 } & Acreedores bancarios & 25 & \\
\cline { 2 - 4 } & Aportaciones de capital & 17 & \\
\cline { 2 - 4 } & No informa & 17 & $92 \%$ \\
\hline
\end{tabular}

Fuente: Elaboración personal del análisis de resultados

El aspecto de finanzas es el de mayor revelación encontrada en los estados contables, dotando de mayor detalle las actividades de operación y de financiamiento que, en suma, otorgan información de operación en un $54 \%$ y $75 \%$ respectivamente.

Este detalle de información es cotidiano como conceptos estándar en los estados contables emitidos en el territorio nacional, sin que las notas de revelación amplíen el concepto de significación.

Con relación a la última variable de información capital humano $y$, en ell, mano de obra no hubo detalle de información, prevaleciendo la falta de información en $37 \%$ y la ausencia de detalle en $62 \%$. Sin que ameritara un detalle de observación.

A partir de los resultados anteriores, se cruzó la información para detectar la correlación de la información, a pesar de los datos encontrados de ausencia y limitación de la revelación informativa de los estados contables, a partir de lo cual la matriz de Spearman arrojó las siguientes correlaciones fuertes: 
- Producción e inventários:

- Compras e inventários: $\quad 0.7143$

- Finanzas e inventários: $\quad 0.6810$

- Finanzas y clientes: $\quad 0.6786$

- Clientes e inventários: $\quad 0.6667$

La correlación entre conceptos se centró en dos ejes básicos: los inventarios y los clientes, desde la función de finanzas; de tal forma que el equilibrio de la información queda cojo, al comparar que el eje de productividad, manufactura y operación, no se correlaciona con alguna otra información en la mayor parte de la muestra.

\section{Conclusiones}

Son diversas las consideraciones teóricas para lograr estudiar con confiabilidad el binomio gestión - contabilidad, dentro de las cuales la posición del investigador y sus propias limitaciones juegan un papel importante, de manera que pueden darse distintas aristas de interpretación de la información contable.

Se aclara que los distintos enfoques de estudio de la gestión y la contabilidad corresponden a economías capitalistas, en un contexto de tecnología de información, modelos y conocimiento contable, y la conexión con inversiones extranjeras.

El estudio del resultado de la gestión a través de los resultados contables forma parte de los paradigmas contables, entrando en conflicto con el paradigma del usuario al no lograr atender las necesidades de verdad y objetividad necesaria para medir la gestión y servir como punto de referencia para la toma de decisiones.

La transparencia es una característica sobre entendida para los resultados contables; sin embargo, es afectada a partir de los grupos directivos de los órganos de gobierno de las organizaciones, al elegir alternativas de registro por conveniencia, llevando con ella a una posible opacidad, simulación e incluso a fraudes

A partir de la capacidad de los sistemas contables para visualizar y estudiar la gestión y la capacidad de actuación de la organización, se encontró que la información cotidiana, emitida públicamente por or- 
ganizaciones económicas industriales en México, no logra informar al usuario general en materia de producción, manufactura y operaciones, no permitiendo detectar la capacidad operativa de la organización y el uso de sus recursos de manufactura, observándose una tendencia a presentar conceptos genéricos que no dan detalle de los resultados sin permitir un análisis segmentado y detallado.

Lo mismo sucede en relación con las ventas y el marketing, que en varios estados contables, se agrupa en conceptos híbridos que permiten dudar acerca de la definición del concepto. Siendo una posición común no dar detalle al respecto.

Con relación al uso y aplicación de recursos, este es más visible en la incidencia de los grupos estándar de los estados financieros como activos y pasivos, siendo un poco débil la descripción de conceptos patrimoniales y de capital social.

Si tomamos en cuenta que estos estados contables estudiados, tienen como objeto hacer una presencia comercial en las páginas web y dar información al usuario social, no necesariamente interno, se detecta el cuidado en la generación de la información y el ocultamiento deliberado de detalle y conceptos que puedas ser mal utilizado. Esto declara una desconfianza de las organizaciones sobre el uso y acceso de su información.

A partir de esto último la transparencia contable, es utilizada como un medio de protagonismo pero no para la toma de decisiones, ni para informar a un usuario general. Presentado una transparencia opaca y manipulada de la gestión de las organizaciones estudiadas.

No fue posible comprobar la hipótesis, quedando esta como un supuesto teórico sujeto a nuevos estudios.

\section{Referencias}

AECA (s/f). La contabilidad de gestión como instrumento de control. Ed. AECA (Colección Documentos). Madrid.

ANTHONY R.N. \& Govindarajan V. (2007). Sistemas de Control de Gestión. (Duodécima edición). Mc Graw Hill. México.

ARGANDOÑA, A. (2007). La responsabilidad social de la empresa a la luz de la ética. Responsabilidad Social Corporativa. ACCID: Barcelona. Pp. 27-37. 
AYUSO, M. Barrachina, M. Garrigos R., Tamari C. \& Urquidi A. (2011). Casos prácticos resueltos de contabilidad de costes. Editorial Profit: Barcelona.

BALADA Ortega, T. \& Ripoll Feliú, V.(2000). Situaciones y tendencias de la Contabilidad de Gestión en el Ámbito Iberoamericano. Ediciones Gráficas Ortega: España.

BRENNAN N. B. \& Solomon J. (2008). Corporate governance, accountability and mechanims of accountability: an overview. Accounting, Auditing \& Accountability Journal. (Vol. 21 No. 7). Pp.885-906.

CHIAVENATO I. (2002). Administración en los nuevos tiempos. Ed. Mc. Graw Hill. Colombia.

CINIF. (2014). Normas de Información Financiera A 1 Estructura de las Normas de Información Financiera. Normas de Información Financiera (NIF). Ed. CINIF, ANFECA, IMCP. Pp. 1-19.

CÓRDOVA Villegas A., Font Playán I., Gudiño Pérez P., Hernández Mendoza E. \& Sánchez Martínez A. (1998). Un enfoque de estudio de la cultura organizacional. Revista Gestión y estrategia. Universidad Autónoma Metropolitana: México. (Núm. 14), (junio-diciembre).

DARMER P. (2000). The subject (ivity) of Management. Journal of Organizational Change Management. (Vol. 13 No. 4). Pp. 334-351.

DE LA ROSA Leal M. E. (2008). La contabilidad de gestión ante la responsabilidad social corporativa. Una propuesta de modelo en el contexto mexicano. Revista Iberoamericana de Contabilidad de Gestión, (Vol. VI, no. 11), (Enero - junio 2008). Pp. 13-41.

DE LA ROSA Leal M.E. (2009). Modelo de costos medioambientales. Metodología y caso: industria maquiladora. Ed. Plaza y Valdez.

DE LA ROSA Leal M.E. \& Gracida Romo J.J. (2008) Cinco décadas de historia colegiada contable en Sonora (1958-2008). Ed. Colegio de Contadores Públicos de Sonora, Universidad de Sonora.

DEL TORO Rovira, R. (2003). Y la historia continúa. Un lustro en la historia de nuestro Instituto, México, Instituto Mexicano de contadores Públicos, A.C.

ESTUPIÑAN Gaitán, R. \& Estupiñán Gaitán, O. (2010). Análisis financiero y de gestión. ECOE Ediciones: Bogotá, Colombia. 
FAYOL H. (s/f). Teoría de Fayol, funciones y principios de administración. Recuperado de http://www.apuntesfacultad.com/teoria-de-fayol-funciones-y-principios-de-administracion.html

FERNÁNDEZ Fernández, A. \& Muñoz Rodríguez, M.C. (1997). Contabilidad de gestión y excelencia empresarial. Editorial Ariel: Barcelona.

GODDARD A. (2004). Budgetary practices and accountability habitus. A grounden theory. Accounting, Auditing \& Accountability Journal. (Vol. 17 No. 4) pp. 543-577.

GINER S., Lamo de Espinosa E. \& Torres C. (2006). Diccionario de Sociología. Ed. Alianza Editorial. Madrid.

GUTIÉRREZ Hidalgo, F. (2005) Evolución histórica de la contabilidad de costes y de gestión (1885-2005). De Computis. Revista Española de Historia de la Contabilidad.

HODGE B.J., Anthony W.P. \& Gales L.A. (1998). Teoría de la Organización. Ed. Prentice Hall (5 a edición). Barcelona.

KOONTZ H. (1994) Elementos de Administración. Mc Graw Hill: México.

KOONTZ, H. \& Weihrich, H. (1994). Administración. Una perspectiva global. (10a ed.), Editorial McGraw-Hill, México.

LÓPEZ y López F. (traductor) (2004). Historia de la Contabilidad. Asociación Española de Contabilidad y Administración de Empresas, Ilustre Colegio Central de Titulados Mercantiles y Empresariales. Madrid.

MOLDOVAN B., Achim S. A. \& Bota Avram C.(2010). Figthing the enemy of fair view principle.Getting to know creative accounting. Anale sthntifice ale Univesitatii. Numar special. Pp: 52-61.

PAHLEN Acuña R.J.M. \& Campo A.M. (2008). Doctrina y Contaminación Ambiental. Contabilidad Ambiental de Gestión y Financiera. (Primera parte). Universidad de Buenos Aires, Facultad de Ciencias Económicas. Pp. 23-41.

PORTER, M. E. (2001). Estrategia competitiva: técnicas para el análisis de los sectores industriales y de la competencia. Compañía Editorial Continental: México.

SEGUNDO V. A. (1998) Aspectos sobre la evaluación de la eficiencia del desarrollo organizacional. Revista Gestión y estrategia. Universidad Autónoma Metropolitana: México. (Núm. 14), (junio - diciembre). 
SHAFRITZ Jay M. \& Steven Ott J. (Compiladores) (2002). The population ecology of Organizations. Classics of Organization Theory. Brooks Cole Publishing Company. (Third Edition). Beltmont, California. Pp. 329-341.

STRAUSS A. \& Corbin J. (1990). Basics of Qualitive Research: Grounded Theory Procedures and Techniques. Sage.

TUA PEREDA, J. (1990). Algunas implicaciones del paradigma de utilidad en la disciplina contable. Revista Contaduría. Universidad de Antioquia. (No.16), (marzo). pp. 17-48

VÁZQUEZ Garatachea E. \& Lozano Avilés B. (1998). Como mejorar la calidad en la empresa: ideas y reflexiones preliminares. Revista Gestión y estrategia. Universidad Autónoma Metropolitana: México. (Núm. 14), (junio - diciembre). 